\title{
Effect of wearing positions of load on the dynamic balance during gait
}

\author{
Seung-Hyun Hyun, Che-Cheong Ryew* \\ Department of Kinesiology, College of Natural Science, Jeju National University, Jeju, Korea
}

The study aim to analyze the influence on dynamic stability relative to positions carrying with weight during gait, for which experiment participated with healthful adult males $(n=9)$, were performed under three-dimensional (3D) conditions of no load (NL), right of trunk (RT), and front of trunk (FT). The kinematic and kinetic conclusions obtained from 3D cinematography and ground reaction force system were as follows; 1-step time elapsed showed longest in NL of all, and maximum velocity of $\mathrm{RT}$ showed slower than $\mathrm{NL}$ and $\mathrm{FT}$, which resulted in significant level at $(P<0.05)$ respectively. While NL showed slower average velocity than RT and FT, RT showed larger incline angle (extrapolated center of mass $\theta)$ than NL and FT, which resulted in significant level at $(P<0.01)$ respectively. Also FT showed larger peak vertical force than $\mathrm{NL}$ and $\mathrm{RT}$, which resulted in significant level at $(P<0.001)$. Because gait pattern is regarded it as a success when fulfilled both forward propulsive force and dynamic stability, we may ensure that each other active strategy for securement and performance in a situation carrying with $20 \mathrm{~kg}$ of the same weight in forward and lateral plane was mobilized. However, participants in pathological gait such as leg injury, and exercise rehabilitation during walking should avoid carrying loads bag to ensure dynamic stability.

Keywords: Gait, Load, Extrapolated center of mass, Center of mass velocity, Dynamic balance

\section{INTRODUCTION}

Postural control is no longer considered simply a summation of static reflexes but, rather, a complex skill based on the interaction of dynamic sensorimotor processes (Horak, 2006). That is, which may be resulted from both being not maintained a perfect balance during bipedal gait of human and positioning correctly on supporting base of center of mass (COM) due to loading of body weight on the other leg (Bauby and Kuo, 2000; Hof et al., 2007).

These unstable systems may be improved by only active control, that is, with considering gravity, condition of supporting area, and visual environment in addition to active alignment of trunk and head (Hof et al., 2007). But locomotion loaded with heavy weight parcel during gait may be seen easily in daily lives, industry fields and various work environments. Particularly gait in various working field was not regarded it as labors, because locomotion was performed with the same weight as body weight in

fact (Hsiang and Chang, 2002). Then, when loaded on body with additional parcel, height of COM of whole body was escalated and imbalance of posture in static and dynamic condition was increased (Singh and Koh, 2009).

Therefore it is necessary to understand biomechanical mechanism on gait control and load transfer among segments in order to improve the dynamic stability during gait with additional weight load and to apply various supporting skill on loads to body according to gait length, load characteristics (i.e., size, weight, location) and the others working environment (Hsiang and Chang, 2002). Like this, the risk factors influencing on instability of gait has been verified, it is very difficult to evaluate and quantify the gait stability.

One of methods which evaluate stability of gait is to grasp an information on stability boundary of gait pattern through perturbing of subject until falling injuries occurred (Hsiang and Chang, 2002). Particularly Hof et al. (2007) reported that one

${ }^{\star}$ Corresponding author: Che-Cheong Ryew (D) http://orcid.org/0000-0001-9473-3990 Department of Kinesiology, College of Natural Science, Jeju National University, 102 Jejudaehak-ro, Jeju 63243, Korea

Tel: +82-64-754-3588, Fax: +82-64-757-1752, E-mail: ryew@jejunu.ac.kr

Received: September 27, 2017 / Accepted: January 7, 2018 
rigid body model concentrated on COM system of inverted pendulum model (IP-Model) maintained the balanced stability by center of pressure (COP) of foot segment, and COM positioned on right foot, the other case also positioned as the same.

That is, gait stability can be maintained by stepping strategy (Hof et al., 2005; Horak and Nashner, 1986), and it may be is critical factor to evaluate a dynamic stability by positioning within vertical projection of COM (Hof et al., 2007). Also Hof et al. (2005) reported that it is necessary to decide a safe area within supporting range with extrapolated center of mass (XCOM) included factors of COM position, eigenfrequency, $\omega_{0}=\sqrt{g / h}$. Because acceleration of COM in inverted pendulum model is proportional relation between COP and distance of projection of $\mathrm{COM}$, it may be insufficient to convert direction of $\mathrm{COM}$ in case of too fast velocity of COM against COP direction (Hof et al., 2007). And then, because gait was performed through anterior-posterior direction mostly, it may more evaluate accurately a gait stability in left-right direction than anterior-posterior direction accustomed already (Chang and Yoon, 2010).

Therefore the study aims to analyze the influence on dynamic stability relative to positions of carrying with weight during gait. For this, we present materials for balancing maintenance of posture and preventing of falling injuries through analysis on gait characteristics (1-step time, velocity, and peak vertical force) calculated by IP-Model and XCOM.

\section{MATERIALS AND METHODS}

\section{Subject}

Adult males skilled in normal gait $(\mathrm{n}=9)$ participated as subject (mean age, $23.00 \pm 1.32$ years; mean heights, $1.73 \pm 0.01 \mathrm{~m}$; mean weights, $72.38 \pm 3.89 \mathrm{~kg}$ ), all of whom understood and consented on the purpose and details of the study.

\section{Experimental procedure}

The 4 ea camera (HDR/HDV 1980i, Sony Corp., Tokyo, Japan) and its light facilities which can capture entire gait motion relative to positions of carrying with weight during gait and 1 system of GRF (AMTI-OR9-7, AMTI, Watertown, MA, USA) were installed at 5-m distance from start on gait path respectively. And then, firstly filmed for $5 \mathrm{sec}$ against control point frame $(2 \mathrm{~m} \times 2$ $\mathrm{m} \times 1 \mathrm{~m}$ ) with 36 landmarks to calculate the 3D spatial coordinates. Reflexive markers (31) of body segment parameter (Plagenhoef et al., 1983) to calculate COM and velocity of whole body for XCOM was attached on the right-left toe, right-left heel, right- left lateral-medial malleolus, right-left shank, right-left lateral-medial epicondyle, right-left thigh, right-left anterior superior iliac spine, sacrum, right-left lateral-medial wrist, right-left lateral-medial elbow, right-left shoulder, chin, and nose, respectively.

Gait was performed orderly from common gait without additional weight with no load (NL) to 20-kg barbell on between right (RT) and front (FT) of trunk loaded with weight. Gait pattern was performed with preferred velocity on bare foot with proper gait pattern. Analysis direction was defined as $y$ axis for proceeding, $x$ axis for lateral for $y$ axis, $z$ axis at vertical direction for $y-x$ axis.

\section{Definition of analysis phase}

Gait analysis was performed at the most increased point of leftright lateral of COM during supporting phase of right foot, and then incline angle of left-right of XCOM-COP (Hof et al., 2007). as follows (Fig. 1).

$$
\begin{aligned}
& \omega_{0}=\sqrt{g / h} \\
& X C O M=x(i)+\left(1 / \omega_{0}\right) \cdot(d x / d t) \\
& X \operatorname{COM} \theta(i)=\tan ^{-1}(X(i)-x 1(i), Z(i)-z 1(i))
\end{aligned}
$$

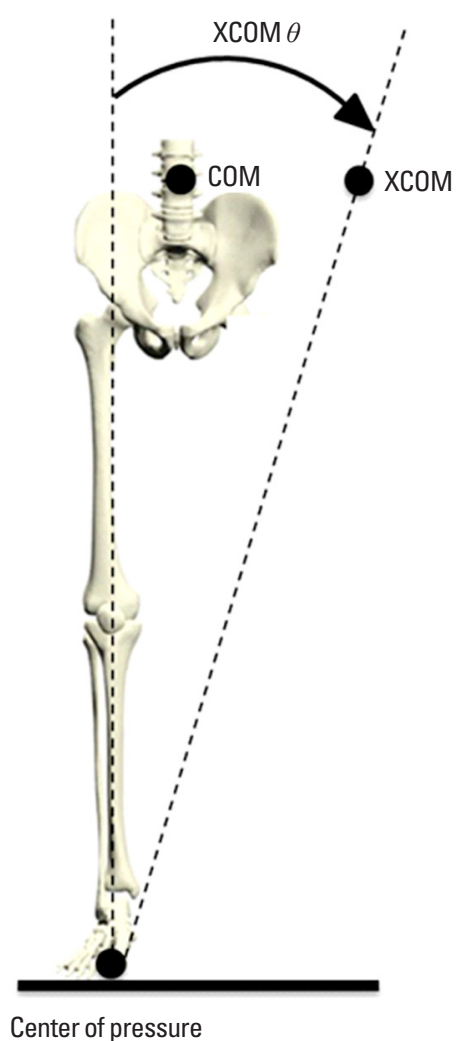

Fig. 1. Extrapolated center of mass (XCOM) during right foot stance. COM, center of mass. 


\section{Nomenclature for XCOM}

$\omega_{0}=$ pendulum eigen frequency

$g=$ acceleration of gravity $9.81 \mathrm{~m} / \mathrm{sec}^{2}$

$b=$ effective height of the body COM above the floor $=1.34 \mathrm{l}$

$x(i)=$ lateral position of $\mathrm{COM}$

$X(i), Z(i)=$ vertical and lateral position of $\mathrm{COM}$

$x(i), z(i)=$ vertical and lateral position of $\mathrm{COP}$

Peak vertical force in addition to XCOM was normalized (N/ $\mathrm{BW}$ ) against maximum value of right supporting phase, and supporting time (sec), maximum velocity, minimum velocity, and mean velocity during 1 step respectively.

\section{Analysis and process of data}

Kwon 3D XP v4.0 (Visol, Gwangmyeong, Korea) was used to process obtain kinematic variables during gait. The kinetic variables were calculated using a control point, body-joint coordinate transformation and tuning, while the 3D coordinates were calculated using the direct linear transformation method. The cutoff frequencies obtained using the Butterworth low-pass filtering method were smoothed at $6 \mathrm{~Hz}$ to remove the noise before using them as data. The average and the standard deviation of the calculated variables were obtained using IBM SPSS Statistics ver. 22.0 (IBM Co., Armonk, NY, USA) and repeated analysis of variance for comparisons among the three gaits $(P<0.05)$.

\section{RESULTS}

Kinematics, XCOM, and peak vertical force (PVF) variables relative to position of carrying with weight during gait are as of (Table 1). The kinematic and kinetic conclusions obtained from 3D cinematography and GRF system were as follows; 1-step time elapsed showed longest in NL of all, and RT showed slower maximum velocity than NL and FT, which resulted in significant level at $(P<0.05)$ respectively. While NL showed slower average velocity than RT and FT, RT showed larger incline angle XCOM $\theta$ than NL and FT, which resulted in significant level at $(P<0.01)$ respectively. Also FT showed larger peak vertical force than NL and RT, which resulted in significant level at $(P<0.001)$.

\section{DISCUSSION}

Gait pattern was regarded it as successful locomotion when fulfilled both forward propulsive force and stability factors (Brunt et al., 1991). Variables related with propulsive force were analyzed for supporting time and velocity of COM. FT showed the shortest supporting time of three kinds of gait, while showed the most early in variables of maximum velocity, average velocity, and minimum velocity of three kinds of gaits.

While 1-step time elapsed showed the longest in NL compared with RT and FT, and showed large difference between maximum velocity and minimum velocity. The result may mean that FT and RT loaded additional weight decreased controlling function of COM velocity when added weight, therefore the gait characteristics resulted in difference according to positions carrying weight, but which may assume to accept and alter a position and velocity of COM, and change of inertia to induce forward movement and gait stability successfully (Pai and Patton, 1997; Redfern and Schumann, 1994).

Particularly FT carried with load on front of trunk more influenced on increase of propulsive force in forward direction, which resulted in increased PVF by instant great momentum generated due to increased velocity of COM and decrease of 1-step time

Table 1. XCOM, peak vertical force, and kinematic variables during walking

\begin{tabular}{|c|c|c|c|c|c|c|}
\hline \multirow{2}{*}{ Section } & \multicolumn{3}{|c|}{ Position of load } & \multirow{2}{*}{$F$} & \multirow{2}{*}{$P$-value } & \multirow{2}{*}{ Posthoc } \\
\hline & No load (NL) & Right of trunk (RT) & Front of trunk (FT) & & & \\
\hline 1-Step time (sec) & $0.55 \pm 0.04$ & $0.54 \pm 0.04$ & $0.53 \pm 0.05$ & 4.846 & $0.021^{*}$ & $\mathrm{NL}>\mathrm{FT}$ \\
\hline \multicolumn{7}{|l|}{ Velocity of COM (m/sec) } \\
\hline Maximum & $1.96 \pm 1.87$ & $1.87 \pm 0.17$ & $2.19 \pm 0.51$ & 3.859 & $0.040^{*}$ & $\mathrm{FT}>\mathrm{NL}>\mathrm{RT}$ \\
\hline Average & $1.53 \pm 0.10$ & $1.64 \pm 0.11$ & $1.65 \pm 0.16$ & 6.629 & $0.007^{* *}$ & $\mathrm{FT}, \mathrm{RT}>\mathrm{NL}$ \\
\hline Minimum & $1.29 \pm 0.24$ & $1.43 \pm 0.11$ & $1.43 \pm 0.08$ & 1.925 & 0.175 & NS \\
\hline ХСОМ $\Theta$ (degree) & $4.14 \pm 2.78$ & $6.70 \pm 1.60$ & $3.38 \pm 1.01$ & 7.890 & $0.003^{* *}$ & $\mathrm{RT}>\mathrm{NL}, \mathrm{FT}$ \\
\hline Peak vertical force (N/BW) & $1.18 \pm 0.08$ & $1.59 \pm 0.08$ & $1.61 \pm 0.15$ & 77.306 & $0.001^{* * *}$ & $\mathrm{FT}>\mathrm{RT}>\mathrm{NL}$ \\
\hline
\end{tabular}

Values are presented as mean \pm standard deviation.

XCOM, extrapolated center of mass; COM, center of mass; NS, not significant.

${ }^{*} P<0.05$. ${ }^{* *} P<0.01$. ${ }^{* *} P<0.001$. 
elapsed. Therefore FT may effects on muscular-skeletal system with great impact force and also increase energy expenditure for velocity control of COM.

IP-Model has been used frequently to characterize a control of static posture, in which defines posture control as relation between COP and COM (Lafond et al., 2004). Though relation between COP and COM is proper means to evaluate a static stability but rather XCOM $\theta$ applied with length of lower leg, velocity of COM, and eigenfrequency, $\omega_{0}=\sqrt{g / l}$ is fittest model to evaluate dynamic stability during gait.

Early studies on dynamic stability with XCOM $\theta$ was performed with patient of knee amputation (Hof et al., 2007), also which was applied to overcome a height obstacle relative to lower length (0\%, 30\%, 40\%, 50\% length of lower leg) (Chang and Yoon, 2010). Chang and Yoon (2010) reported that XCOM $\theta$ was 8.59 degree during daily gait and 9.93 degree of maximum height of obstacle, while XCOM $\theta$ of this study was 4.14 degree of NL, 6.70 degree of RT and 3.38 degree of FT respectively.

Although showed incline angle of less range when compared with previous study, RT showed characteristics of more inclined angle than NL and FT among three kinds of gait. COG of body moves forwardly during one leg supporting in a view point IP-model (Hof et al., 2007), but considerable change of COM position and velocity in case of carrying on lateral load of trunk may assume to influence on a dynamic stability. While XCOM more reduced pattern in FT than NL, which may assume to be result controlled against a right-left lateral movement of COM by function controlling velocity to secure dynamic stability.

Rehabilitation of locomotion has been considered to be unattainable following a clinically complete or severely incomplete spinal cord injury even after conventional therapy. However, the locomotion of spinal animals can be improved by training that provides complex temporal patterns of sensory information related with stepping that is interpreted by the spinal cord (Harkema, 2001).

The materials on gait characteristics obtained various weight and carrying positions on body may be helpful for efficient gait and prevention of secondary risk of falling injuries. Though carrying whether anterior or lateral plane of body with the same $20 \mathrm{~kg}$ of weight, It was assumed that human uses the other active strategy for performance and securement of dynamic stability and propulsive force. However, participants in pathological gait such as the leg injury, and exercise rehabilitation during walking should avoid carrying loads bag to ensure dynamic stability.

\section{CONFLICT OF INTEREST}

No potential conflict of interest relevant to this article was reported.

\section{REFERENCES}

Bauby CE, Kuo AD. Active control of lateral balance in human walking. J Biomech 2000:33:1433-1440.

Brunt D, Lafferty MJ, Mckeon A, Goode B, Mulhausen C, Polk P. Invariant characteristics of gait initiation. Am J Phys Med Rehabil 1991;70: 206-212.

Chang JK, Yoon SH. Evaluation of gait stability using medio-lateral inclination angle in male adults. Korean J Sport Biomech 2010;20:261-266.

Harkema SJ. Neural plasticity after human spinal cord injury: application of locomotor training to the rehabilitation of walking. Neuroscientist 2001;7:455-468

Hof AL, Gazendam MG, Sinke WE. The condition for dynamic stability. J Biomech 2005;38:1-8.

Hof AL, van Bockel RM, Schoppen T, Postema K. Control of lateral balance in walking. Experimental findings in normal subjects and aboveknee amputees. Gait Posture 2007;25:250-258.

Horak FB. Postural orientation and equilibrium: what do we need to know about neural control of balance to prevent falls? Age Ageing 2006;35 Suppl 2:ii7-ii11.

Horak FB, Nashner LM. Central programming of postural movements: adaptation to altered support-surface configurations. J Neurophysiol 1986;55:1369-1381.

Hsiang SM, Chang C. The effect of gait speed and load carrying on the reliability of ground reaction forces. Safety Sci 2002;40:639-657.

Lafond D, Duarte M, Prince F. Comparison of three methods to estimate the center of mass during balance assessment. J Biomech 2004;37:14211426.

Pai YC, Patton J. Center of mass velocity-position predictions for balance control. J Biomech 1997;30:347-354.

Plagenhoef S, Evans FG, Abdelnour T. Anatomical data for analyzing human motion. Res Q Exerc Sport 1983;54:632-635.

Redfern MS, Schumann T. A model of foot placement during gait. J Biomech 1994;27:1339-1346.

Singh T, Koh M. Effects of backpack load position on spatiotemporal parameters and trunk forward lean. Gait Posture 2009;29:49-53. 